\title{
Effects of mating dynamics and crowding on sex ratio variance in mice
}

\author{
S. Krackow* \\ Forschungsinstitut für die Biologie landwirtschaftlicher Nutztiere, Forschungsbereich Populationsbiologie und \\ Züchtungsforschung, Wilhelm-Stahl-Allee 2, 18196 Dummerstorf-Rostock, Germany
}

\begin{abstract}
Mating units of six virgin females and one adult stud male were established to test for the effects of timing of mating and crowding of pregnant females on litter sex ratios in mice. Females either copulated during periods when no other female of the mating unit copulated simultaneously (single mating condition) or when more than one female copulated (multiple matings condition). Two crowding conditions were imposed on the animals: the females of 14 mating units were placed into individual cages after mating (isolated condition), while females of the other 13 mating units remained in the original group until shortly before littering (crowded condition). Sex ratio variance did not deviate from random expectation in litters arising from the multiple matings periods. However, in litters arising from single mating periods, extreme sex ratios were found significantly less frequently than expected by chance. Higher sex ratio variance in litters arising from multiple matings periods is attributed to the timing of mating being at higher variance under this condition, which is known to affect sex ratios in other rodents. Crowding significantly reduced sex ratio variance further. Reduced sex ratio variance under single mating and crowded conditions is speculated to follow from competition for resources between preimplantation embryos, which may be further increased by stressful effects of crowding. Loss of embryos after implantation appeared not to be responsible for the above effects.
\end{abstract}

\section{Introduction}

Sex ratio biases have frequently been reported among rodents and other mammals (Parkes, 1926; Clutton-Brock and lason, 1986; James, 1987; Cockburn, 1990; Hiraiwa-Hasegawa, 1993; Hardy, in press), and there appears to be a considerable potential for the mother to manipulate the sex ratio at birth (Krackow, 1995a). However, up to now there has been no convincing evidence for a physiological cause of the observed variations, although three mechanisms have received closer attention in sex ratio studies over the past two decades (reviewed by Krackow, 1995b). First, timing of copulation and insemination within the oestrous cycle of the female have repeatedly been shown to influence the sex ratio at birth in rodents (Hedricks and McClintock, 1990; Huck et al., 1990; Hornig and McClintock, 1994; Hornig and McClintock, 1996). Second, gonadotrophin and steroid hormone concentrations appear to be linked with the sex ratio in many studies and may mediate sex ratio skews in relation to dominance rank and other psychological stressors (James, 1989, 1996). Third, fetal resorption has occasionally been found to be sex-specific (Gosling, 1986; Pratt et al., 1989; Krackow, 1990; Krackow, 1992).

In this study, a simple experimental paradigm was constructed to test simultaneously for the effects of the timing of mating and of crowding of females (as a potential psycho-

\footnotetext{
*Present address: Abteilung Sinnesbiologie, Institut für Biologie, Humboldt Universität, Invalidenstr. 43, 10115 Berlin, Germany.

Revised manuscript received 17 February 1997.
}

logical stressor) on the sex ratio in mice. The mating dynamics were varied by establishing mating units of one adult stud male and six virgin females. Owing to the Whitten-effect, matings were expected to cluster at day 3 after cohabitation, increasing the probability of several females being in oestrus concurrently. After mating, females were either kept under crowded conditions by leaving them in the original mating cages until shortly before littering, or they were kept individually.

\section{Materials and Methods}

\section{Experimental procedure}

The mice were from an outbred control line kept at the Forschungsinstitut für die Biologie landwirtschaftlicher Nutztiere. For the isolated condition, 1.4 mating units of six virgin females and one male were established in perspex Macrolon cages $(26.5 \mathrm{~cm} \times 42 \mathrm{~cm} \times 15 \mathrm{~cm})$. Females were checked for vaginal plugs each morning and evening for 6 days. When a plug was found, or on day 6 after cohabitation with the male, females were transferred to individual cages $(22 \mathrm{~cm} \times 36 \mathrm{~cm} \times 15 \mathrm{~cm})$. For the crowded condition, 13 units of six females and one male were again established $(n=76$; two females, from different groups, were accidentally lost). Vaginal plugs were checked for as in the isolated females, but females were kept together until 18 days after introduction of the male, when they were placed into individual cages. The males were always removed on day 6 after cohabitation. 


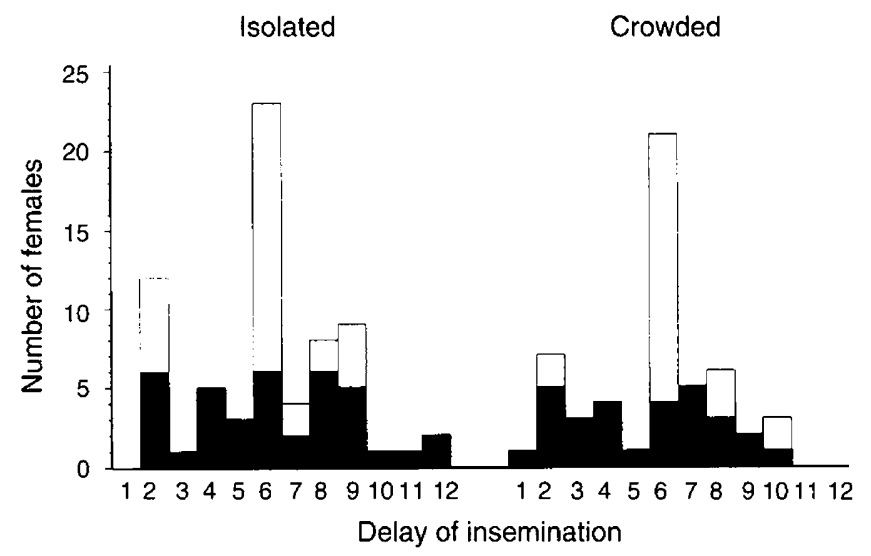

Fig. 1. Distribution of matings over the 12 half-day periods after establishment of mating units, for crowded and isolated females under single mating condition ( $\mathbf{\square}$ ) and multiple matings condition $(\square)$.

Consequently, there were 12 possible periods of insemination. In the case of fertile females of which a plug was missed at inspection (26 of 109 fertile females), the half day of conception was estimated by subtracting 19 days (the modal duration of gestation in fertile females with vaginal plugs) from the time of parturition. In 13 of the 51 females that did not produce a litter, a vaginal plug had been found. The total number of inseminations (number of conceptions plus number of infertile females showing plugs) was considered to be the number of matings per period. For analysis, litters were classed as having been conceived during periods of either a single female (single mating condition) or more than one (range 2-5) female (multiple matings condition) having been inseminated. The distribution of matings under both conditions over half-day periods is given for crowded and isolated females, respectively (Fig. 1).

Females were checked twice per day for litters. At birth, pups were sexed by means of the anogenital distance and the maternal uteri were dissected. Uterine scars were classed as being either yellow or red (Krackow, 1992). Yellow scars represent implantation sites of embryos that have died early in utero, whereas the difference between the number of red scars and the number of pups at birth may represent late fetal mortality or pups lost as a result of infanticide. Females that did not produce litters were also dissected and their uteri searched for remnants of gestation.

\section{Statistical procedures}

The effects of crowding, mating condition and their interaction upon sex ratios (proportion of males) as well as on early and late fetal loss were tested using generalized linear modelling. This allowed exploration of effects on the binomially distributed numbers of males or lost embryos (events) given the respective litter size or number of scars (trials) using logits as a link function and a likelihood ratio test for significance of effects (SAS Institute Inc., 1993). Effects of covariates, such as number of uterine scars (that is, litter size at implantation), litter size at birth, and amount of fetal loss on the sex ratio, were tested by the same procedure. Generalized model ANOVAs were used for tests of treatment effects on the number of uterine scars and litter size.

If sex were determined randomly at fertilization and no sex ratio-specific loss of zygotes or embryos occurred afterwards, the number of males at each litter size would follow a binomial distribution; that is, sex ratio variance would fit the binomial expectation. Since litter size varies, it is not straightforward to test for deviation from this random expectation. Deviation of the sex ratio variance from binomial expectation was tested using the statistic suggested by James (1975), which is independent of litter size effects on the mean sex ratio and of any overall skew of the sex ratio. The measure of the deviation of the sex ratio variance from the binomial expectation is the dispersion of the litter gender distribution. This dispersion $(K: I)$ and its variance (1:I) were calculated from

$$
\begin{gathered}
K=\sum_{i=1}^{N} 0.5\left(f(f-1): q^{2}+m(m-1): p^{2}+2 f m: p q\right) \\
I=\sum_{i=1}^{N} n(n-1): 2 p^{2} q^{2}
\end{gathered}
$$

where $m=$ number of males, $n=$ litter size, $\mathrm{f}=n-m$, $N=$ number of litters, $p=$ proportion of males, $q=1-p$. The standard normal deviate could then be derived as $z=|(K: I)|$ $\sqrt{ }(1: I) \mid$, to test for significance of the deviations (see Krackow, 1992, for a more detailed account). The dispersion measure yields negative values if the distribution is under-dispersed (sub-binomial; that is, sex ratios are less variable than expected by binomial chance), and yields positive values if the distribution is over-dispersed (super-binomial; that is, sex ratios are more variable than expected by chance).

Fisher's exact probability test was applied to contingency table data. Two-sided probabilities are given, and were considered significant at $P<0.05$.

\section{Results}

Significantly more isolated (64 of 84) than crowded females (45 of 76) produced litters (Fisher's test, $\mathrm{df}=1, P<0.03$ ). Of the fertile females, 35 isolated and 24 crowded females were inseminated under single mating conditions. None of the infertile females showed signs of implantation. There were no significant effects of crowding, mating condition, or their interaction, respectively, upon the sex ratio, litter size at birth or implantation, or the amount of early and late fetal loss $(P>0.1$ in all cases; Table 1$)$. Mean sex ratios were also not affected by the number of uterine scars, litter size, or the amount of early, late, and total fetal loss $(P>0.1$ in all cases).

Variance of sex ratios in litters of isolated dams did not differ significantly from that expected under the binomial model, but was significantly lower than expected in litters of crowded dams (Table 2). When grouped according to mating condition, variances appeared to be lower than expected in litters conceived during single mating periods (Table 2). Exclusion of all litters that suffered from fetal loss did not alter these relationships: sex ratios had significantly sub-binomial variance in the crowded condition $(n=16, K: I=-0.0181, z=2.39$, $P<0.02)$ but not in litters from isolated dams $(n=22$, 
Table 1. Basic statistics of reproductive parameters for the experimental groups

\begin{tabular}{|c|c|c|c|c|c|c|}
\hline & \multicolumn{3}{|c|}{ Isolated } & \multicolumn{3}{|c|}{ Crowded } \\
\hline & $n$ & Mean & SD & $n$ & Mean & SD \\
\hline \multicolumn{7}{|l|}{ Single mating } \\
\hline Uterine scars & 35 & 13.14 & 2.61 & 24 & 12.04 & 3.62 \\
\hline Litter size & 35 & 11.94 & 2.59 & 24 & 11.08 & 3.88 \\
\hline Late fetal loss & 35 & 0.91 & 1.36 & 24 & 0.58 & 0.83 \\
\hline Early fetal loss & 35 & 0.29 & 0.46 & 24 & 0.38 & 0.65 \\
\hline Sex ratio (proportion of males) & 35 & 0.54 & 0.17 & $23^{*}$ & 0.51 & 0.16 \\
\hline \multicolumn{7}{|l|}{ Multiple matings } \\
\hline Uterine scars & 29 & 13.10 & 2.38 & 21 & 12.14 & 4.47 \\
\hline Litter size & 29 & 11.66 & 2.69 & 21 & 10.86 & 3.88 \\
\hline Late fetal loss & 29 & 1.17 & 1.47 & 21 & 1.00 & 1.10 \\
\hline Early fetal loss & 29 & 0.28 & 0.59 & 21 & 0.29 & 0.56 \\
\hline Sex ratio (proportion of males) & $28^{*}$ & 0.49 & 0.15 & 21 & 0.49 & 0.18 \\
\hline
\end{tabular}

*Pups of two litters were destroyed by their mothers and could not be sexed.

Table 2. Dispersion of the litter gender distribution (K:I), its variance ( $1: I)$, the standard normal deviate $(z)$ and the associated two-sided probabilities $(P)$ for litters of crowded and isolated females, conceived during single and multiple mating periods

\begin{tabular}{lccccc}
\hline & $n$ & $K: I\left(\times 10^{2}\right)$ & $1: I\left(\times 10^{4}\right)$ & $z$ & $P$ \\
\hline Single mating & 57 & -0.8208 & 0.1577 & 2.07 & $<0.04$ \\
Multiple matings & 49 & -0.5077 & 0.1884 & 1.17 & ns \\
Isolated & 63 & 0.1987 & 0.1423 & 0.53 & ns \\
Crowded & 43 & -1.2798 & 0.2323 & 2.66 & $<0.008$ \\
\hline
\end{tabular}

$\mathrm{ns}$, not significant.

$K: I=-0.0031, z=0.53$, not significant), and sex ratio variance was lower than expected in litters from single mating periods $(n=21, K: I=-0.0122, z=1.94, P=0.052)$ in contrast to those from multiple matings periods $(n=17, K: I=-0.0038$, $z=0.57$, not significant). Furthermore, crowding reduced sex ratio variance under each of the two mating conditions. In litters of isolated females, the variance of sex ratios was not significantly sub-binomial under either matings condition (single mating: $n=35, K: I=-0.0081, z=1.71,0.05<P<0.1$; multiple matings: $n=28, K: I=-0.0032, z=0.58$, not significant) but sex ratio variance was significantly sub-binomial in litters of crowded females (single mating: $n=22$, $K: I=-0.0177, z=2.64, P<0.01$; multiple matings: $n=21$, $K: I=-0.0130, z=1.97, P<0.05)$.

\section{Discussion}

In multiple matings periods, mating dynamics are inevitably more variable than in single mating periods since the males must share copulatory sequences among females (in singlemating settings, sequences last for $1-5 \mathrm{~h}$ from the onset of copulation at about 03:00 h; S. Krackow, unpublished data on II copulatory sequences; see also Dewsbury, 1972). If the timing of mating had an effect on the litter sex ratios, as is found in other laboratory rodents, this would have led to sex ratios being distorted towards having a male bias in some litters, and towards having a female bias in others. The increase of sex ratio variance (that is, an increase in the frequency of extreme sex ratios) in litters from multiple matings periods compared with those from single mating periods indicates that mating dynamics also affect litter sex ratios in mice.

Reduced sex ratio variance in litters conceived during single mating periods is as yet unexplained. One hypothesis for sub-binomial variation supposes that the probability of becoming male or female varies for zygotes formed at different stages of the fertilization period (Brooks et al., 1991). However, the second major finding of the current study is that crowding further reduced variation of the sex ratio, which requires an additional explanation. Another recent hypothesis may accommodate both findings. This hypothesis holds that whenever there is intrasexual competition for resources among blastocysts, or resources for development are limiting during a specific period of blastocyst development, sub-binomial variation of the sex ratio could result (Krackow, in press). Hence, any baseline competition between blastocysts would lead to reduced variation in sex ratio in the absence of any varianceincreasing factor; this would be in agreement with the reduced variance displayed under the single mating condition. An increase in the strength of competition within litters (that is, the 
increased social stress of the crowded condition) would then be expected to reduce further the litter sex ratio variance. However, as yet there is no direct evidence for either of the above processes.

The results of the present study indicate that crowding of females reduced fertility. This is in agreement with previous findings that crowding can result in longer periods of anoestrus or pseudopregnancy (De Catanzaro and MacNiven, 1992). Since some infertile females possessed vaginal plugs during the mating period, total implantation failure may also have contributed to infertility.

Finally, as the effects of experimental treatment on sex ratio variance were apparent even when all litters affected by fetal loss were excluded, the events affecting sex ratio variance in this study must have taken place before implantation, or at least too early to yield signs of implantation at birth.

The author is indebted to P. S. Burgoyne, B. Puppe, and two anonymous reviewers for providing highly appreciated comments on the manuscript. The author also thanks I. C. W. Hardy for reading the manuscript and improving the English.

\section{References}

Brooks RJ, James WH and Gray E (1991) Modelling sub-binomial variation in the frequency of sex combinations in litters of pigs Biometrics 47 403-417

Clutton-Brock TH and lason GR (1986) Sex ratio variation in mammals The Quarterly Review of Biology 61 339-374

Cockburn A (1990) Sex-ratio variation in marsupials Australian Journal of Zoology $37 \quad 467-479$

De Catanzaro D and MacNiven E (1992) Psychogenic pregnancy disruptions in mammals Neuroscience and Biobehavioral Reviews 16 43-53

Dewsbury DA (1972) Patterns of copulatory behavior in male mammals The Quarterly Review of Biology 47 1-33

Gosling LM (1986) Selective abortion of entire litters in the coypu: adaptive control of offspring production in relation to quality The American Naturalist $127 \quad 772-795$
Hardy ICW Possible factors influencing vertebrate sex ratios: an introductory overview Applied Animal Behaviour Science (in press)

Hedricks C and McClintock MK (1990) Timing of insemination is correlated with the secondary sex ratio of Norway rats Physiology and Behavior $\mathbf{4 8}$ $625-632$

Hiraiwa-Hasegawa M (1993) Skewed birth sex ratios in primates - should high-ranking mothers have daughters or sons? Trends in Ecology and Evolution $8395-400$

Hornig LE and McClintock MK (1994) Unmasking sex-ratio biasing through targeted analysis Animal Behaviour 47 1224-1226

Hornig LE and McClintock MK (1996) Male sexual rest affects litter sex ratio of newborn Norway rats Animal Behaviour 51 991-1005

Huck UW, Seger J and Lisk RD (1990) Litter sex ratios in the golden hamster vary with time of mating and litter size and are not binomially distributed Behavioral Ecology and Sociobiology 26 99-109

James WH (1975) The distribution of the combinations of the sexes in mammalian litters Genetical Research, Cambridge 26 45-53

James WH (1987) The human sex ratio. Part I: a review of the literature Human Biology 59 721-752

James WH (1989) Parental hormone levels and mammalian sex ratios at birth Joumal of Theoretical Biology 139 59-67

James WH (1996) Evidence that mammalian sex ratios at birth are partially controlled by parental hormone levels at the time of conception Journal of Theoretical Biology 180 271-286

Krackow S (1990) Sex-specific embryonic mortality during concurrent pregnancy and lactation in house mice Journal of Experimental Zoology 256 106-112

Krackow S (1992) Sex ratio manipulation in wild house mice: the effect of fetal resorption in relation to the mode of reproduction Biology of Reproduction 47 $541-548$

Krackow S (1995a) Potential mechanisms for sex ratio adjustment in mammals and birds Biological Reviews, Cambridge 72 225-241

Krackow S (1995b) The developmental asynchrony hypothesis for sex ratio manipulation Journal of Theoretical Biology 176 273-280

Krackow S Further evaluation of the developmental asynchrony hypothesis of sex ratio variation Applied Animal Behaviour Science (in press)

Parkes AS (1926) The mammalian sex-ratio Biological Reviews, Cambridge 2 1-51

Pratt NC, Huck UW and Lisk RD (1989) Do pregnant hamsters react to stress by producing fewer males? Animal Behaviour 37 155-157

SAS Institute Inc. (1993) SAS Technical Report P-243 SAS Institute Inc., Cary, $\mathrm{NC}$ 